\title{
Embedding Domain Discretization for Coupled Topology and Shape Optimization
}

\author{
Gabriel Stankiewicz*, Chaitanya Dev ${ }^{\dagger}$ and Prof. Dr. Paul Steinmann ${ }^{\dagger}$ \\ * Institute of Applied Mechanics (LTM) \\ Friedrich-Alexander Universität Erlangen-Nürnberg \\ Egerlandstrasse 5, 91058, Erlangen, Germany \\ e-mail: gabriel.stankiewicz@fau.de, web page: https://www.ltm.tf.fau.eu/ \\ $\dagger$ Institute of Applied Mechanics (LTM) \\ Friedrich-Alexander Universität Erlangen-Nürnberg \\ Egerlandstrasse 5, 91058, Erlangen, Germany \\ e-mail: paul.steinmann@fau.de, web page: https://www.ltm.tf.fau.eu/
}

\begin{abstract}
Density-based topology optimization and node-based shape optimization are often used sequentially to generate production-ready designs. In this presentation, we address the challenge to couple topology and shape optimization into a single optimization problem by using an embedding domain discretization technique. In this technique, the outline of the embedded body, defined by a $d-1$ dimensional mesh, segregates the elements of the embedding domain into inner, boundary and outer collection. The boundary elements require a special integration approach, which involves oversampling of the integration points to approximate the geometry of embedded body.

In our approach, a variable shape is explicitly represented by the boundary of an embedded body. Large design changes of the shape are easy to handle, since no morphing of the interior of the embedded body is necessary. Hence, complex regularization and remeshing schemes which are used in standard node-based shape optimization methods are not required. Furthermore, the embedding domain in form of a structured mesh allows us to introduce a variable, pseudo density field. This provides a full flexibility in handling topological changes. In regions that reach zero pseudo density, the algorithm introduces additional shape variables.

In this novel approach we couple topology and shape optimization by using an embedding domain discretization. This way, we bring the advantages of both methods together and provide an efficient way to design fine-tuned structures without predefined topological features.
\end{abstract}

